\title{
Application of advanced engineering methods in studying a road traffic event between a 12-wheeler truck and a small tourism in a local junction from Cluj-Napoca
}

\author{
Adela-Ioana BORZAN, Doru-Laurean BĂLDEAN* \\ Technical University of Cluj-Napoca, Romania \\ ${ }^{*}$ Corresponding author: doru.baldean@auto.utcluj.ro \\ Article history \\ Received 10.06.2017 \\ Accepted 23.09.2017 \\ DOI https://doi.org/10.26825/bup.ar.2017.002
}

\begin{abstract}
Road traffic and transportation are some of the most important factors that influence the social and economic contemporary life. Developing new systems and technologies for traffic control and transportation respectively all their auxiliary components is difficult without considering computer aided processing power in testing and evaluation. Main objective of the present paper is to point out the new and advanced method in car crash evaluation of two vehicles caught in a specific traffic event from ClujNapoca. Specific objectives are a theoretical study of the idea for advanced engineering method in car crash evaluation and an applied research developed in the Automotive Laboratory at Technical University from Cluj-Napoca with the motor vehicles implied in road event. Different distractions and inattention situations can transform in catastrophes for truck drivers, particularly with unexpected stoppages in traffic occur. Collisions warning with emergency brake for years now, helps road traffic participants in preventing rear-end collisions by regaining and refocusing their attention to the road and vehicles ahead of them. Thus, when a critical event is detected, a series of collision warnings and signs prompt the driver to react, and if the driver still fails to react, the emergency brakes make sure that a fatal collision is prevented anyway. Beside what already is on the spot present paper proposes an integrated complex system of communication and data transfer between multiple parts and layers involved in road traffic events. The concept and drawings are shown in the paper. Practical measurements were also considered.
\end{abstract}

\section{Introduction}

Drivers may fail to sufficiently increase safety margins to allow time to respond to possible unpredictable events (e.g., lead vehicle braking). Advanced driver assistance systems should 
facilitate and possibly boost drivers' self-regulating behavior. For instance, they might recognize when appropriate adaptive behavior is missing and advice or alert accordingly. The results from this study could also inspire training programs for novice drivers, or locally classify roads in terms of the risk associated with secondary task engagement while driving [1].

Established in 1997, the European New Car Assessment Programme (Euro NCAP) provides consumers with a safety performance assessment for the majority of the most popular cars in Europe. Thanks to its rigorous crash tests, Euro NCAP has rapidly become an important driver safety improvement to new cars. After ten years of rating vehicles, Euro NCAP felt that a change was necessary to stay in tune with rapidly emerging driver assistance and crash avoidance systems and to respond to shifting priorities in road safety. A new overall rating system was introduced that combines the most important aspects of vehicle safety under a single star rating. The overall rating system has allowed Euro NCAP to continue to push for better fitment and higher performance for vehicles sold on the European market. In the coming years, the safety rating is expected to play an important role in the support of the roll-out of highly automated vehicles [2].

The protection of children in motor vehicle crashes has improved since the introduction of child restraint systems. However, motor vehicle crashes remain one of the top leading causes of death for children. Today, computer-aided engineering is an essential part of vehicle development and it is anticipated that safety assessments will increasingly rely on simulations [2].

In this paper is analyzed the concept of telecommunication feature which may be implemented in road traffic between vehicles and a headquarter in order to facilitate fast transferring information or event data to emergency units, police, firefighter, employer company, home and family members (or close relatives). In order to make this study a particular event was analyzed and proper system design was developed. Thus a Dacia Logan was under the research scope, a common vehicle in Romania and EU, which in this particular case was involved in a road traffic event with an 12 wheeler truck few weeks ago (April 2017). Before the crash the vehicle looked as in the Figure 1.

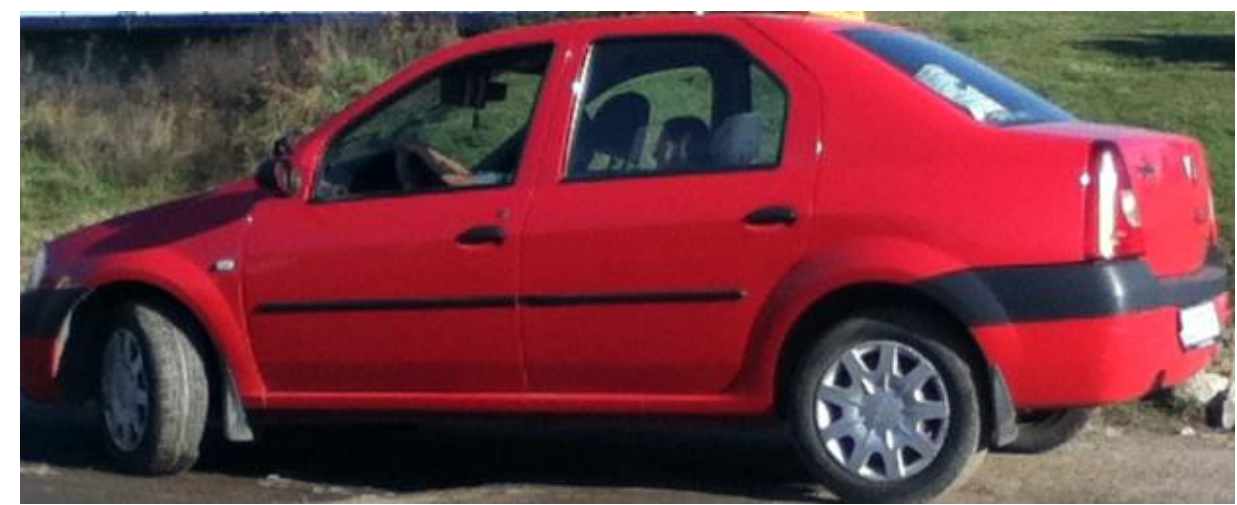

Figure 1. The studied vehicle type model Dacia Logan before the road-traffic event.

Inattention or distraction is a growing concern in the traffic environment and loss of forward road vision in combination with an unexpected event will normally result in an accident. Collision Warning with EMERGENCY BRAKING brings support to the driver in these critical situations by helping to focus the driver's attention towards the traffic in front of the vehicle. If a critical situation is detected, for example, slow or stopped traffic ahead, the Head Up Display on the windshield is be illuminated. If there is no reaction from the driver the Head Up Display starts to flash, supported by a loud audible alarm. If still no reaction from the driver the trucks brakes are applied with a low braking force. Finally if still no reaction from the driver is detected an emergency full-braking is performed. By supporting the truck driver in being aware of the traffic situation ahead, many accidents can be avoided. In order to alert other drivers of panic braking situations the brake lights on the truck will change from fixed to flashing, when extreme brake retardation is called for either by the driver or by the Collision Warning system [4].

If the 12 wheeler truck would be equipped with the auxiliary system of "Collision Warning with Emergency Brake (CW-EB)" most probably the road event would not be taken place. Anyway, in 
this particular context there was not a CW-EB system installed on board and vehicles collided with each other. Figure 2 shows the end result of the road traffic event after the tourism was rear hit by a twelve wheeler truck. The event was generated when the tourism's driver hit the brakes before cornering to the left toward a smaller street. The truck driver wrongly appreciated the situation and didn't hit the brakes so the result was.

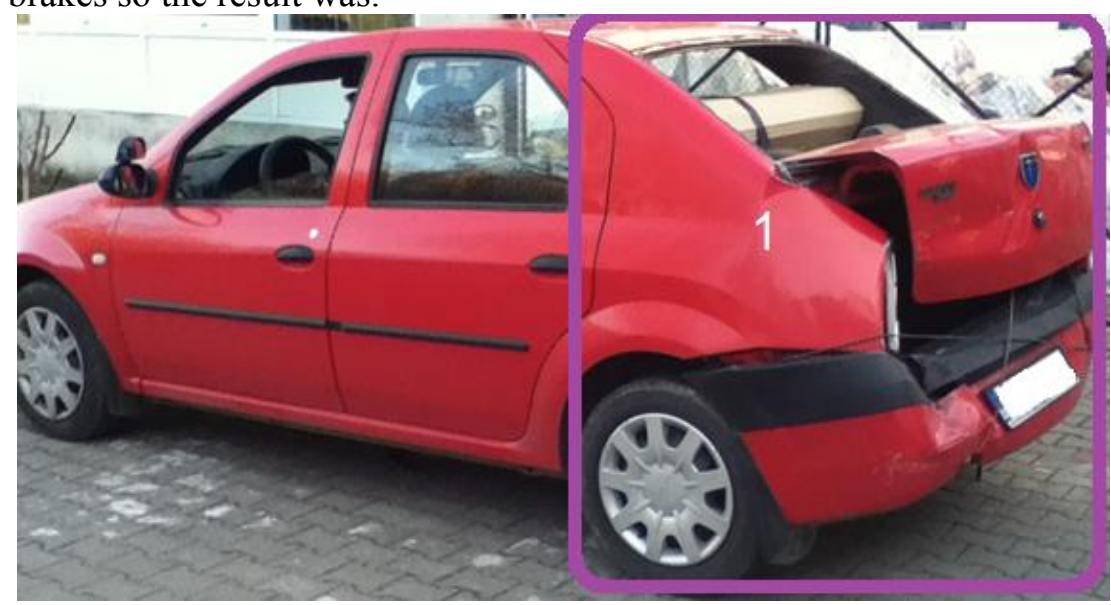

Figure 2. Vehicle after the road-traffic event. 1 -crashed area.

There were young people and children involved in the car crash. That was the main reason for sketching out a protocol for fast data transfer and proper analyzes in order to provide the interested parts (authorities and families) with adequate information about the road event.

In figure 3 is shown the Volvo concept of CW-EB in operation on a virtual model.

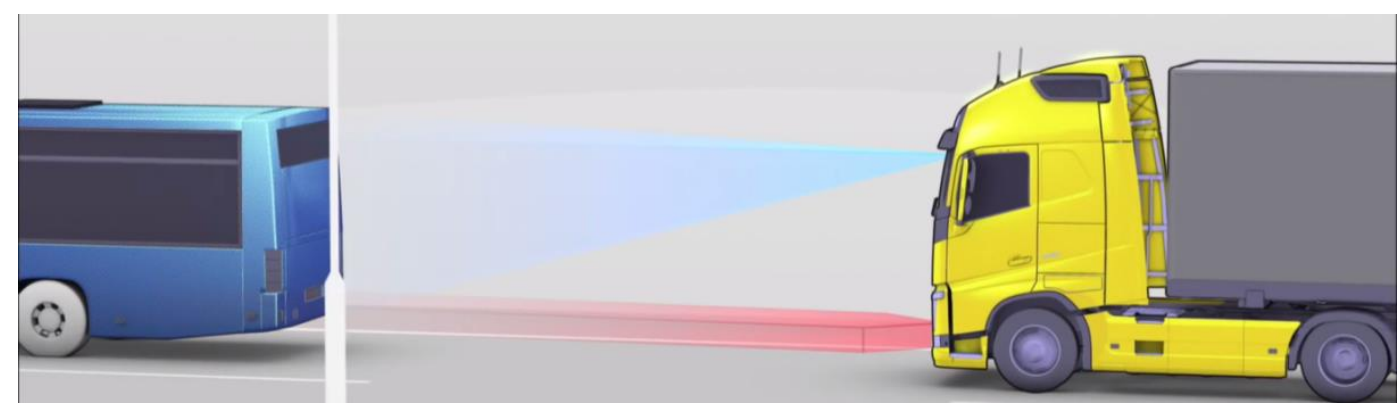

a

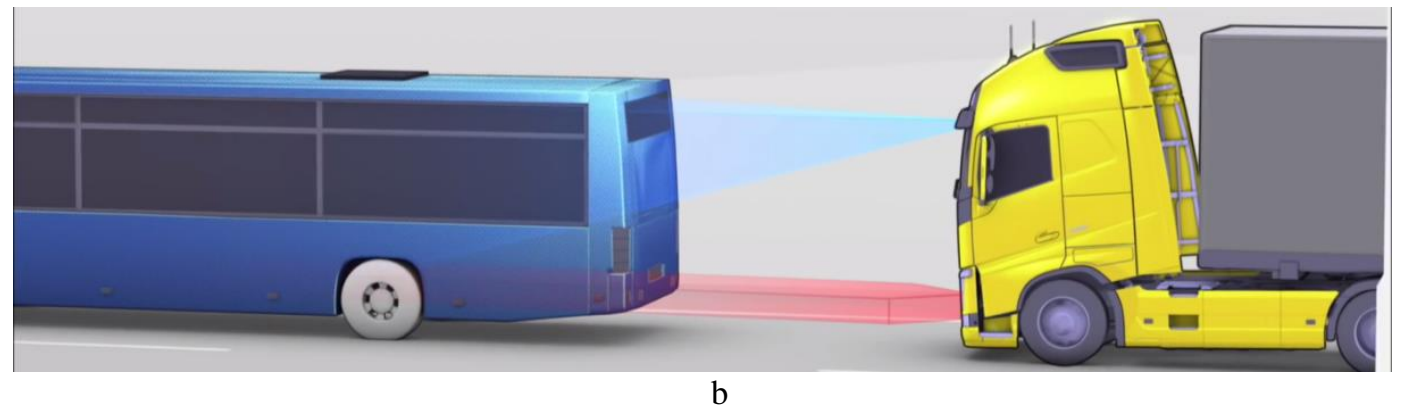

Figure 3. CW-EB from Volvo Trucks working in order to prevent a collision [4]. a-start of detection and anticipation of the road traffic risk ahead; b-emergency brake activation after the expected driver's response was not received by the management system in order to prevent the road hazard.

The present paper shows the possibility of implementing a telecom concept in automotive products in order to improve the data transfer procedure and fast sending information to those that have an interest in the event. 


\section{Methodology and materials}

\subsection{Methodology}

By measuring the deformations, considering the materials and components that were crushed in the impact, respectively by using the computer aided evaluation, it may be assessed and interpreted the damage level. The present work highlights an engineering method for computer aided car crash evaluation and appreciation in the field of material damages concerning the road events in real time traffic. The studied and evaluated event is a real traffic accident which took place in Cluj-Napoca, and was analyzed with accurate means by use of latest technology. A new method of advanced engineering research was put into practice. The relation between computer processing power and car-crash evaluation effort is significantly underlined.

\subsection{Materials}

The present work makes use of two motored vehicles available and involved in road traffic event in order to analyze the causes of the problem and development possibilities for securing future life. Also there were used devices and equipment in order to improve data communication between the involved parties (road traffic participants, police, insurance companies, transport companies and owners) and authorities for a better solving of legal aspects, repairing process and financial aspects.

Figure 5 shows the rear part of the tourism involved in the road traffic event and odometer recorded data, which in some cases is important information. The materials used for making road traffic safer consists in mobile information storage and fast connections to a world wide web or a data transfer network, event documentation and damage appreciation. All of these and the specific components involved are considered in the process of implementing the fundamental idea of this work.

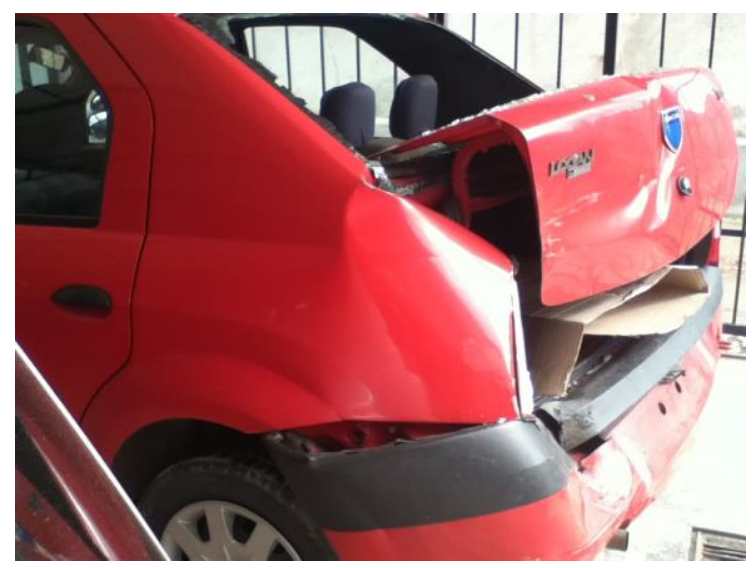

a

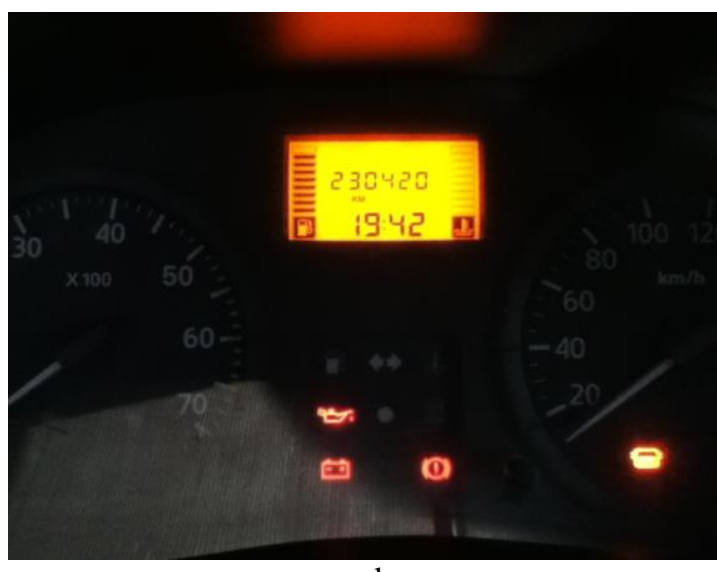

b

Figure 5. The motor vehicle crashed at the rear end by the 12 wheeler. a-rear end of the vehicle after the crash; b-odometer actual value.

\subsection{Mathematics and calculus}

Inertia is the property of all material things especially that makes them to oppose any change in their change of motion-state. Galileo Galilei defined the concept of inertia in the late 1500s. Almost a hundred years after Galileo introduced the concept of inertia, Newton used it to formulate his first law of motion - the Law of Inertia. The rapid transfer of kinetic energy is the cause of crash injuries, so managing kinetic energy is what keeping people safe in car crashes is all about. Crash worthiness is a term used to describe the protection a car offers an occupant in a crash and involves the structure, the restraint system, etc. Knowing the mass of the vehicle and the speed there may be determined the momentum and the force during the collision.

A more accurate definition is represented in the following relation [5]: 


$$
\mathrm{F}=\frac{\mathrm{m} \Delta \mathrm{v}}{\mathrm{t}}
$$

where: $\mathrm{F}$ is force, in $\mathrm{N} ; \mathrm{m}$ - mass, in $\mathrm{kg} ; \Delta$ - change in velocity, in $\mathrm{m} / \mathrm{s} ; \mathrm{t}-$ time, in sec.

The impulse of the collision is expressed in the following relation [5]:

$$
\mathrm{Ft}=\mathrm{m} \Delta \mathrm{v}
$$

where: $\mathrm{Ft}$ is impulse, in $\mathrm{N}^{*} \mathrm{sec} ; \mathrm{m} \Delta-$ change in momentum.

In the present case the truck travels with $\left(\mathrm{v}_{\mathrm{tr}}\right) 50 \mathrm{~km} / \mathrm{h}$ (which is $13.89 \mathrm{~m} / \mathrm{s}$ ) and has a mass $\left(\mathrm{m}_{\mathrm{tr}}\right)$ of $30000 \mathrm{~kg}$, while the tourism precedes the truck with $\left(\mathrm{v}_{\mathrm{to}}\right) 5 \mathrm{~km} / \mathrm{h}(1.389 \mathrm{~m} / \mathrm{s})$ and a mass $\left(\mathrm{m}_{\mathrm{to}}\right)$ of $1.1 \mathrm{t}$ :

$$
\text { Momentum } \Leftrightarrow \mathrm{M}_{\mathrm{x}}=\left\{\begin{array}{l}
\mathrm{M}_{\mathrm{tr}}=\mathrm{m}_{\mathrm{tr}} \cdot \mathrm{v}_{\mathrm{tr}}=30000 \cdot 13.89=416700 \mathrm{~kg} * \mathrm{~m} / \mathrm{s} * 1 \mathrm{sec} \cong 4087827 \mathrm{Nm}, \\
\mathrm{M}_{\mathrm{to}}=\mathrm{m}_{\mathrm{to}} \cdot \mathrm{v}_{\mathrm{to}}=1100 \cdot 1.39=1527.8 \mathrm{~kg} * \mathrm{~m} / \mathrm{s} * 1 \mathrm{sec} \cong 14987.72 \mathrm{Nm} .
\end{array}\right.
$$

Making the transformations it may be observed that the truck momentum during the collision is around $4.08 \mathrm{MN} / \mathrm{m} / \mathrm{s}$. These parameters may be calculated very easily and rapidly by the vehicle control unit (VCU) and initiate a data transfer when the distance is too little and the speed high or when the crash risk increases (or accident already took place).

Using mobile devices to get some access level on the Internet has become lately a common daily activity for many people. Information is transferred and consumed every time a mobile data user is connected to the Internet, whether for manipulating data, sending or receiving emails, downloading or simply browsing web pages [6]. In this way the data transfer can be modelled and tested practically.

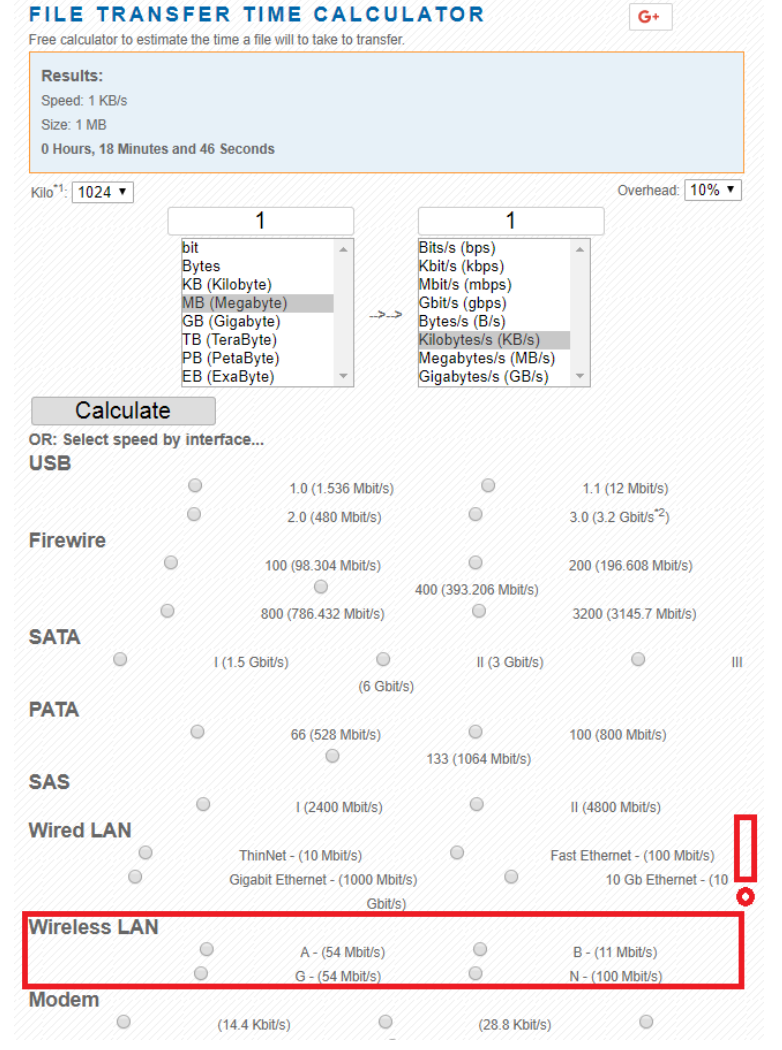

Figure 6. Calculus protocols of the data transfer rate

(a) via different connections [7] and speeds before/after the crash of the vehicles (b) based on known existing models [8]

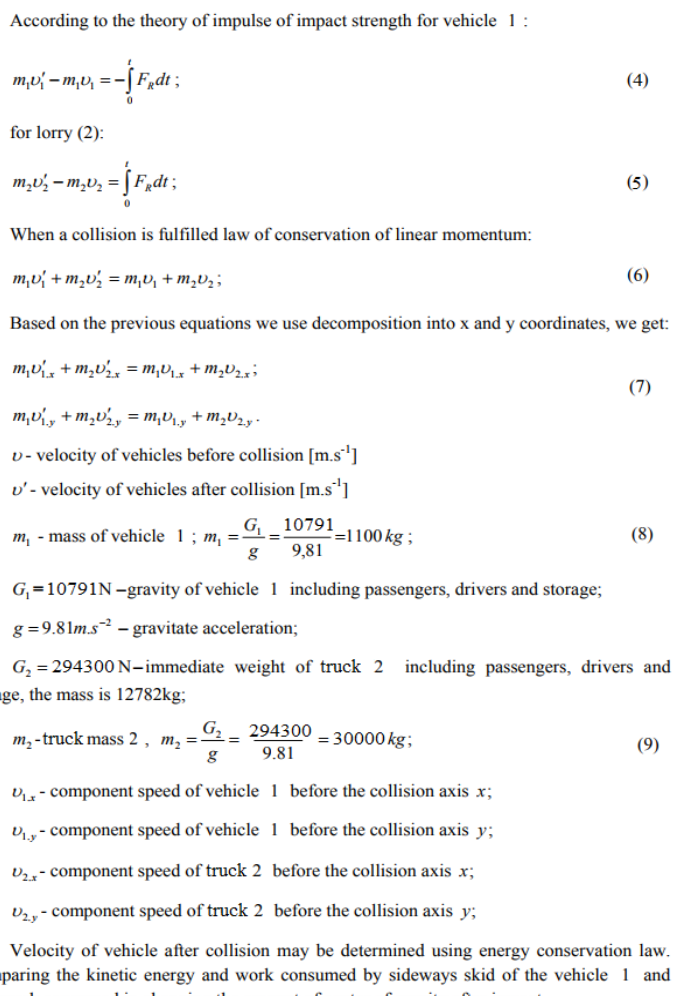
Comparing the kinetic energy and work consumed by sideways skid of the vehicle 1 and also work consumed in changing the amount of center of gravity after impact. 


\section{Experimental setup and protocols}

\subsection{Experimental setup for testing and measurements}

In order to achieve the expected experimental results and to develop some interesting conclusions in relation with the application of advanced engineering methods in studying a road traffic event between a 12-wheeler truck and a small tourism in a local junction from Cluj-Napoca there were designed and implemented specialized devices as shown in figure 7 and figure 8 .
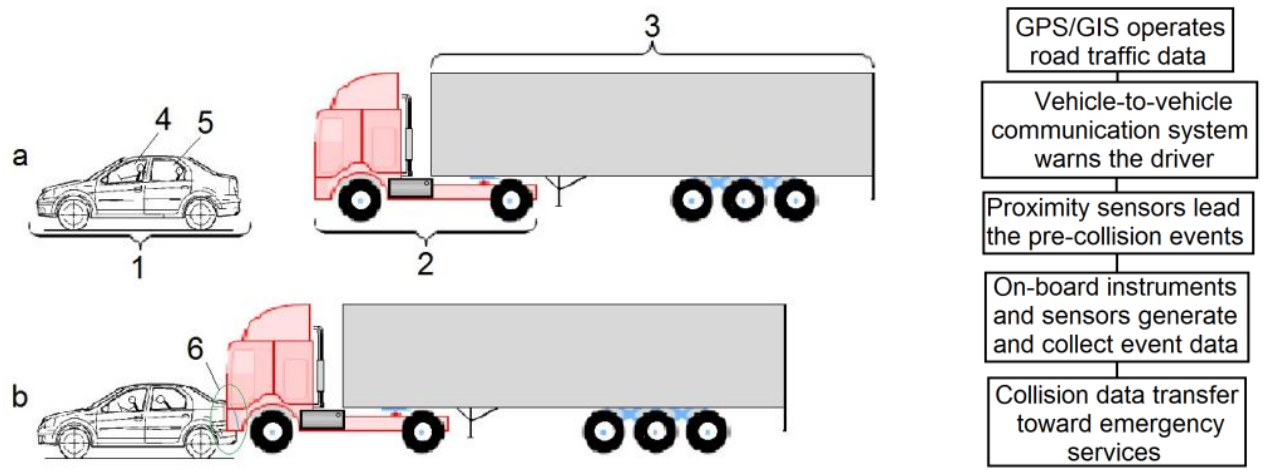

Figure 7. The sequences of the road traffic event

a-prior car crash; b-collision; 1-tourism; 2-semi-truck; 3-trailer; 4-driver (and probably the front occupants); 5-rear seat occupants (other passengers).
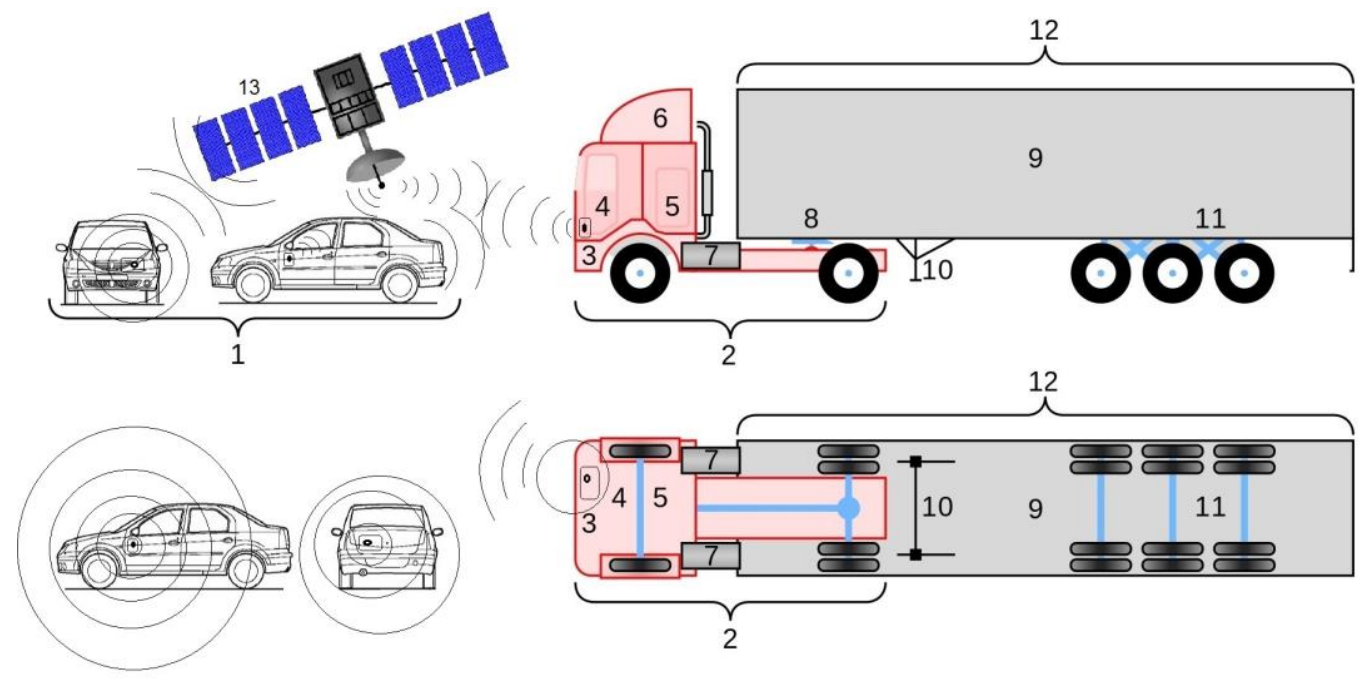

Figure 8. Experimental test equipment for improving the safety standards in road traffic

1-specialized equipped vehicle with VCU; 2-semi-truck with CW-EB and satellite communication capabilities; 3-receiver-transmitter via Wi-Fi connection; 4-driver location in the cabin; 5-resting space; 6-wind shield over the cabin; 7-fuel tank; 8-mechanical connection between the semi-truck and trailer; 9-storage space of the trailer; 10-supporting structure for trailer; 11-rear axles of trailer; 12-trailer; 13-satelit connection facility.

Deformations and vehicle body displacements may be determined specifically in each particular place and case after the vehicles are stabilized as it is shown in figure 9. Also a method of calculating the deformation taking into consideration the mass and energy conservation law in order to have an immediate estimation of the damage on site of the event and to send the data immediately to those who may have some concerns about it. The calculation of crush energy (and equivalent speed) is done by modelling the damage area into crush zones and then determining the energy needed to cause the damage in each zone. The intrusion into each zone, called the crush depth, is measured by a strict protocol that is consistent with the measurements made during the original staged crash tests [9]. 


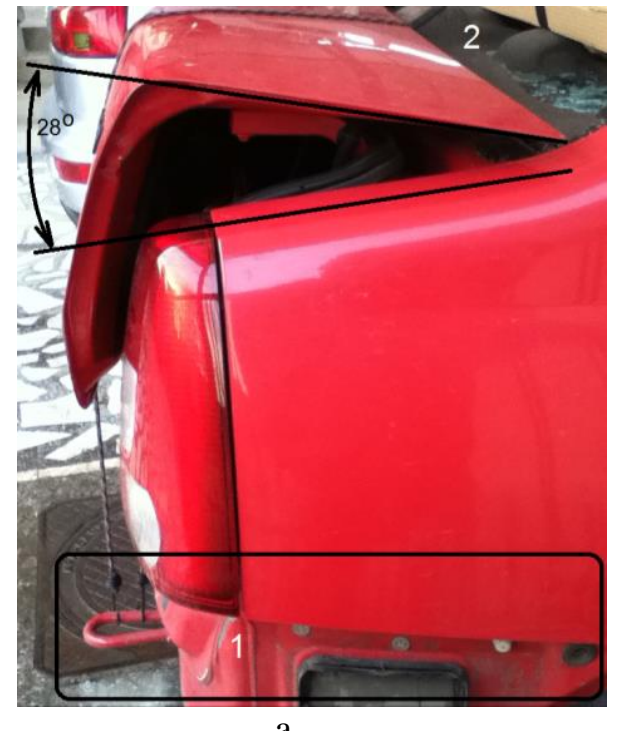

a

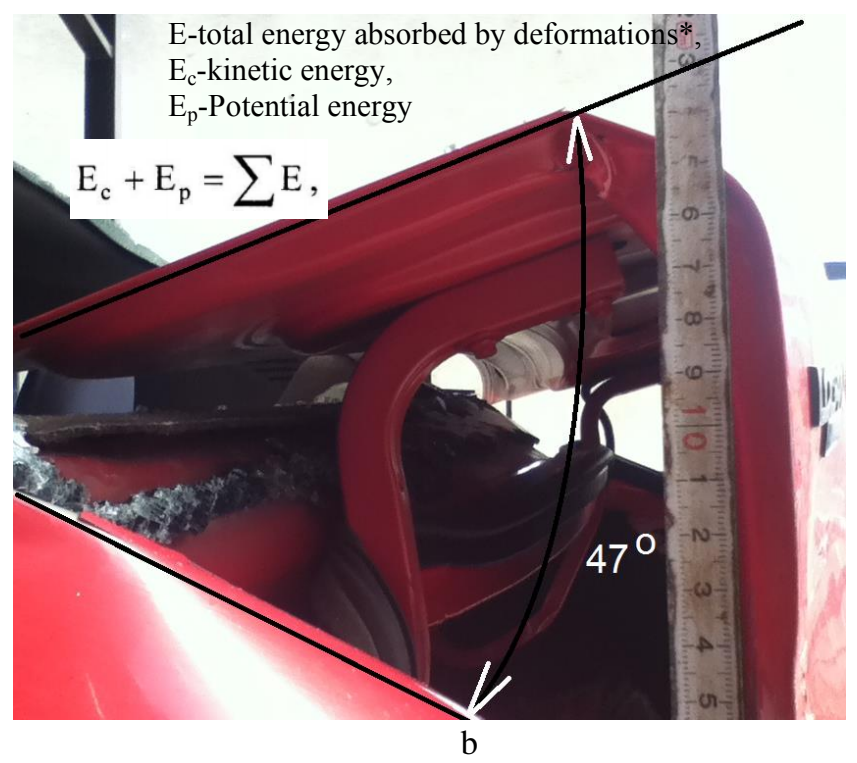

Figure 9. Measuring the deformations of the body components.

a-rear-right side of the crashed vehicle; b-rear-left side with a greater deformation of the components; 1 -lower damaged area; 2-uper area (rear window); *speed during collision is determined considering deformations

[10].

\subsection{Experimental testing protocol}

By defining up a computer assisted communication model and determining the primary components which may give consistent signals of damage, as well as taking into consideration the cables and electric signals it may be generated the data encoding and transfer protocol. In the present paper there is presented an experimental first stage of the system implementation realized till now by defining the necessary apparatus and communications protocols in order to gain access to information on crash site, as shown in figure 10. The vehicle considered is presented in table 1.

The light bulbs if deteriorated present some damaged signals when OBD2 protocol is applied so that signals, as well as the broken cables signals and door open/damaged signals may be encoded in a short message for emergency call or via Wi-Fi communication protocol in order to send the best and appropriate info to whom it may concern.

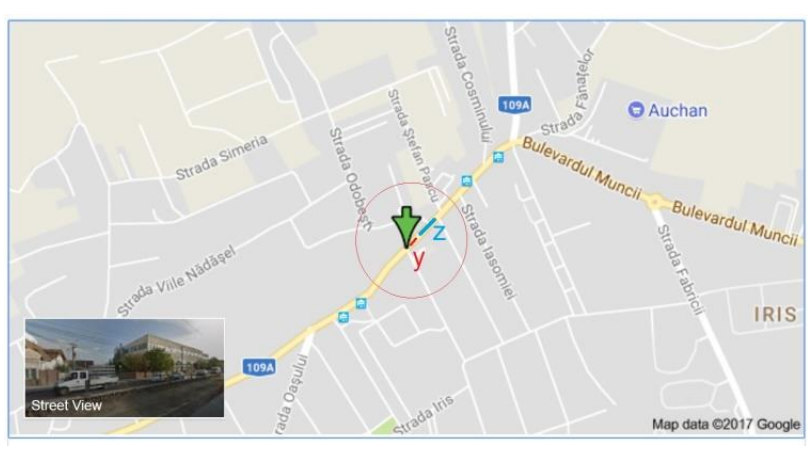

a

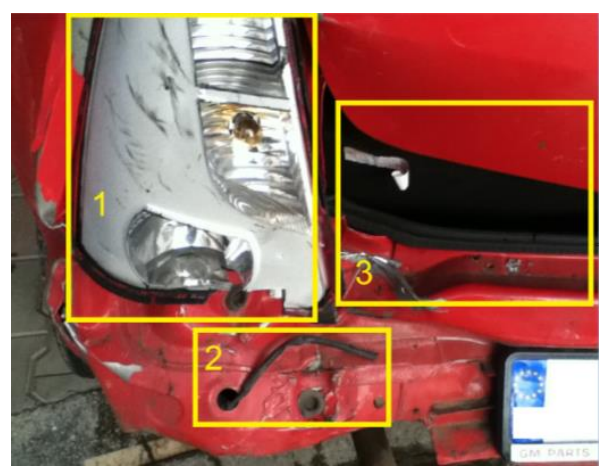

$\mathrm{b}$

Figure 10. Crash site map and significant components that generate signals and data which may be considered in communication process.

a-site map; b-crashed vehicle; y-tourism on site; z-truck on map; 1-light body destroyed; 2-cable interrupted; 3-cargo door displaced. 
Table 1. Technical specs of tested tourism.

\begin{tabular}{ll}
\hline Parameter & Actual Value \\
\hline Manufacturer & Dacia \\
Model & Logan \\
Engine displacement & $1500 \mathrm{~cm}^{3}$ \\
Fuel type & Diesel \\
Particular features & Engine Control Unit, ABS C.U., Central C.U. \\
Maximum speed & $160 \mathrm{~km} / \mathrm{h}$ \\
Number of standard control units / diagnosis method & $3 / \mathrm{OBD} 2$
\end{tabular}

\subsection{Limitations of the proposed study}

The research is limited to the presentation and application of the new engineering method in computer aided car-crash evaluation for a specific road event with two motor vehicles, but it may be applied in complexes cases and specific situations also. The case study and method will be further improved.

\subsection{The novelty of the achievement}

The authors gained experience in manufacturing engineering, rapid prototyping, alternative technologies in manufacturing and computer aided technologies as well as in automotive engineering. The consideration of car crash evaluation aspects in road traffic conditions represents an extension of research capabilities and interdisciplinary experience. Even if there were also studied in the previous works some particular dynamic tests of vehicles, this is the first integrating research of so many important aspects that define road events evaluation and advanced engineering methods based on computer processing power available at Technical University from Cluj-Napoca.

\section{Conclusions}

Redefining the methods in safety and transportation is close related to the car-crash and road events research. Crash parameters are not manifesting alone as sole factors in conservative environment. A road event should be considered in relation with the complex traffic whole which is dynamic environment. The car crash aspects have a major influence in the vehicles and traffic development and their operation or correlation, but in the same time both of them (vehicle and the system) have a secondary financial impact when it comes to human society. Development possibilities and trend lines are also shown in order to lead out the further studies on the proposed subject and to effectively create a safer road traffic environment. The proposed idea transforms the motored vehicles in data storage devices and routers in a vast information network that has secure dynamic transfers as the main objective of its existence. They even may determine by themselves degree of deformations and speeds.

\section{References}

[1] Tivestena E, Dozza M, 2015, Driving context influences drivers' decision to engage in visual-manual phone tasks: Evidence from a naturalistic driving study Journal of Safety Research vol 53 pp 87-96

[2] Ratingen M et al, 2016, The European New Car Assessment Programme: A historical review Chinese Journal of Traumatology vol 192 pp 63-69

[3] Brolin K, 2015, Safety of children in cars: A review of biomechanical aspects and human body models IATSS Research vol 382 pp 92-102

[4] *** 2012 Volvo Trucks - Collision Warning with Emergency Brake http://www.volvotrucks.com/

[5] Jones G, 2000, Understanding Car Crashes: It's Basic Physics! Insurance Institute for Highway Safety https://education.ufl.edu/gjones/files/2013/04/teachers_guidePhysics.pdf

[6] *** 2012 Check Data http://www.mobilenet.gov.hk/en/consumer_tips/data_usage_calculator /index.html

[7] *** 2014 File transfer time calculator https://techinternets.com/copy_calc?do, 2017.07.23

[8] *** Bayarjargal Tseveennamjil 2011 Determining the speed of vehicles before and after crash http://pernerscontacts.upce.cz/22_2011/Tseveennamjil.pdf, 2017.08.23

[9] *** Crash Reconstruction Basics for Prosecutors http://www.ndaa.org/, 2017.08.23

[10] Todoruţ A 2008 Dinamica Accidentelor de Circulaţie UTPress Cluj-Napoca pp. 99-124 